This item was submitted to Loughborough's Research Repository by the author.

Items in Figshare are protected by copyright, with all rights reserved, unless otherwise indicated.

\title{
Resilience in the blood transfusion process: Everyday and long-term adaptations to 'normal' work
}

PLEASE CITE THE PUBLISHED VERSION

https://doi.org/10.1016/j.ssci.2019.07.028

PUBLISHER

Elsevier BV

VERSION

AM (Accepted Manuscript)

\section{PUBLISHER STATEMENT}

This paper was accepted for publication in the journal Safety Science and the definitive published version is available at https://doi.org/10.1016/j.ssci.2019.07.028

LICENCE

CC BY-NC-ND 4.0

\section{REPOSITORY RECORD}

Watt, Alison, Gyuchan Thomas Jun, and Patrick Waterson. 2019. "Resilience in the Blood Transfusion Process: Everyday and Long-term Adaptations to 'normal' Work”. figshare.

https://hdl.handle.net/2134/9227033.v1. 


\title{
Resilience in the Blood Transfusion Process: Everyday and Long-Term Adaptations to 'Normal' Work
}

\author{
Alison Watt a,b*, Gyuchan Thomas Jun ${ }^{\mathrm{a}}$, Patrick Waterson ${ }^{\mathrm{a}}$ \\ aHuman Factors \& Complex Systems Group, Loughborough Design School, \\ UK \\ bSerious Hazards of Transfusion (SHOT), NHS Blood \& Transplant, \\ Manchester, UK \\ *Corresponding author: Loughborough Design School, Loughborough \\ University, Epinal Way Loughborough Leicestershire LE11 3TU.
}

A.Watt@lboro.ac.uk

\begin{abstract}
Blood transfusion is considered one of the safer aspects of healthcare, however potentially avoidable patient-safety incidents led to 14 deaths in the United Kingdom in 2017. Improvement initiatives often focus on staff compliance with standard operating procedures, which fail to understand adaptations made in a complex, dynamic environment. Therefore, the aim of this study is to examine the extent and nature of adaptations at all stages of the full vein to vein transfusion process. Thirty-seven employees described sixty-six adaptations in their transfusion practices, showing clear differences between what has been characterised as work-as-imagined (WAI) and workas-done (WAD). An analysis of the adaptations using the Systems Engineering Initiative for Patient Safety 2.0 (SEIPS 2.0) shows that triggers for adaptations were mostly staff-related or driven by poor information technology systems, but the resultant adaptations were usually amendments to tasks and processes. The majority of adaptations (83\%) were forced - ideal solutions are not possible, so workarounds and coping strategies are required, but some $(17 \%)$ were proactive - the surrounding system is adequate, but performance is improved by adapting. Managers or colleagues were largely unaware of the adaptations made (79\%) and, as a result, opportunities may be missed to identify and learn from resilient practices. The Concepts for Applying Resilience Engineering (CARE) model was further articulated in order to shed
\end{abstract}


extra light on triggers and mechanisms. We make a number of suggestions regarding how we can better learn from adaptations and how these could be used to improve the safety of the blood transfusion process.

Keywords: Blood Transfusion; Healthcare; Resilience; Adaptation; Systems Engineering Initiative for Patient Safety 2.0 (SEIPS 2.0); Concepts for Applying Resilience Engineering (CARE) 


\section{Introduction - Human Factors/Ergonomics and healthcare}

Over the course of the last decade or so the field of human factors and ergonomics has witnessed huge expansion within healthcare and patient safety. Research involving assessments of safety climate and culture, team working arrangements and the role played by situation awareness in healthcare delivery have become common and continue to expand in interest and application [Waterson and Catchpole, 2016; Carayon et al., 2014; Carayon, 2011]. In 2013 NHS England developed a concordat agreed by many major NHS bodies that signed up to commit their organisations to integrate a wider understanding of Human Factors and Ergonomics (HFE) principles to improve their patient safety work [NHS England, 2013]. Health Education England (HEE), which is a signatory to the concordat, has incorporated HFE into the education and training for patient safety. HEE recently published a report which recommended that "[the] principles of Human Factors and professionalism must be embedded across education and training" [Commission on Education and Training for Patient Safety, 2016].

Other recent initiatives have maintained support for the importance of HFE in healthcare, such as the formation of the Healthcare Safety Investigation Branch [HSIB], whose investigation model for serious healthcare incidents is based on past research in safety science and Human Factors [HSIB, 2018, p.2]. The Care Quality Commission, which is the independent regulator of health and social care in England, has encouraged a systems approach to quality improvement in hospitals [CQC, 2018, p34]. In October 2018 the General Medical Council (GMC) announced a plan to roll out Human Factors training to all their fitness to practise decision makers, case examiners and clinical experts [GMC, 2018] with the aim of embedding Human Factors into their processes when investigating adverse events and serious failings.

\subsection{Human Factors and blood transfusion}

One area which has recently been the subject of study by Human Factors experts is blood transfusion. Much of the process of blood transfusion has been considered to be an area of highly standardised care and the approach to management of risk in transfusion is categorised as ultra safe and avoiding 
risk [Vincent and Amalberti, 2016, page 29]. However, there have also been serious historical risks with transfusion [Infected Blood Inquiry, 2019] and death is still the outcome of a handful of transfusions each year. Fourteen potentially avoidable deaths for example, occurred in 2017 [Bolton-Maggs et al., 2018]. Our earlier research involved analysing transfusion safety incidents [Anonymised] and concluded that there was a tendency to focus on blaming individual staff members for incidents instead of looking for other, systemrelated, causes of adverse events. As a result, the study described in this paper was carried out in order to examine the normal transfusion process and analyse what individuals do when things go wrong.

\subsection{Adaptations and the transfusion process}

Historically there has only been limited research using Human Factors and Ergonomic principles to improve the safety of the blood transfusion process. Two recent studies used the Functional Resonance Analysis Method (FRAM) [Hollnagel and Goteman, 2004; Hollnagel, 2012] to analyse incidents in transfusion where errors have or could have caused harm. Both studies showed adaptations were common in the transfusion process. Nakajima [2015] investigated two cases of patients receiving blood components intended for another individual and used FRAM to highlight the differences between 'work as imagined' and 'work as done' [Hollnagel, 2015] in the two incidents. Clinicians were forced to make routine adjustments because technology did not always work or was not well understood alongside other adaptations caused by situational pressures and uncertainties. Nakajima concluded that information technology can be one of the most influential factors on performance variability. Pickup et al. [2017] also used FRAM, alongside the Systems Engineering Initiative for Patient Safety (SEIPS), which was used to code observations. FRAM was used to understand why variability can occur in the blood sampling task of the transfusion process. Their conclusions provided insights into why healthcare practitioners may adapt their practice to balance patient safety in the context of fluctuating demands and challenging work environments and equipment e.g. problems with reliability and availability of technology affected the task of taking a blood sample, so staff would adapt their work practices, such as adjusting the 
sequence of the order in which they undertook various tasks when blood sampling or they might distribute different tasks within a team to ensure patient care was not delayed. The current study is slight different in emphasis and focuses on improving the audit process and expanding our understanding and awareness of the key role played by adaptations throughout the entire transfusion process. By comparison, the FRAM studies were narrower and looked at areas of transfusion practice known to be at risk of failure. The present study aimed to tease out gaps in the whole process using a simple question, in order that organisations can learn from this, without necessarily using FRAM. We plan to extend the work described here into an ongoing national audit process known as the 'Vein to Vein Audit' (V2V). It is anticipated that transfusion safety can be improved by encouraging local audit of adaptations and redesign of the process.

Within the Human Factors/Ergonomics literature, work process adaptations have often been cast in a negative light. The literature on violations for example [e.g., Reason et al., 1998] often views non-conformity to rules and procedures as something that should be designed out. By contrast, more recent work, particularly within resilience engineering, treats adaptations as inevitable and sometimes necessary adaptations to local circumstances. According to Safety-II thinking [Hollnagel, 2014; Sujan et al. 2019], safety is defined as an 'ability to make dynamic trade-offs and adjust performance in order to meet changing demands and to deal with disturbances and surprises' [Sujan et al., 2017]. These sorts of dynamic trades-offs involving risk are an intrinsic part of blood transfusion, for example when dangerous reactions or viral infections are clearly outweighed if a patient has a clinical need for replacement blood components. However, achieving this type of risk-benefit balance is sometimes difficult to manage and it is classified as an adverse incident if patients are given a transfusion when lower risk treatments are available, because the level of inherent risk means transfusion should be the last option considered, e.g. iron replacement therapy for anaemia of dietary origin is a much safer alternative than transfusion. These and other patientsafety incidents related to blood transfusion are reportable to the United Kingdom (UK) haemovigilance scheme, Serious Hazards of Transfusion 
(SHOT). Cumulative figures extracted from Annual SHOT Reports published over the period 1996 to 2017 show there have been 262 transfusionassociated deaths and a further 1456 patients have suffered major morbidity [SHOT, 1996-2017].

\subsection{Study aims}

System redesign and improvement within the transfusion process may be aided by increased knowledge of the drivers, means and experience of adaptations being made at all steps of the procedure. The current study adopts a Safety-II perspective, which emphasises how work is achieved, rather than how it is imagined [Hollnagel, 2014]. Safety-II reflects the ability to succeed under varying conditions, so we examined how the systems involved in transfusion are being adapted. We described changes and amendments made at all stages of the full vein to vein transfusion process (i.e. the complete process covering all the steps commencing with taking a sample from a patient's vein through the processes in between, before eventual transfusion of a blood component back into the intended patient's vein).

The Systems Engineering Initiative for Patient Safety 2.0 model (SEIPS 2.0) [Holden et al., 2013b] was chosen for analysis in this study, because this model provides a healthcare-specific framework for the analysis of the sociotechnical work system. It has been widely applied in healthcare [Carayon et al., 2014] including complex surgery [Acher et al., 2015] and primary care [Wooldridge et al., 2017] and even blood sampling [Pickup et al., 2017]. We aim to analyse adaptations using this model to identify areas of weakness or strength throughout the transfusion process.

\section{Methods of study}

\subsection{Setting and participants}

The full vein to vein transfusion process was explored in three UK hospitals, two in Greater London and one in the Midlands, as part of their regular internal audit process. All were large teaching hospitals providing major acute care services and the full nine steps (Figure 1) of the transfusion process 
were carried out in each of these institutions. The study was carried out by staff representing both SHOT and the National Comparative Audit of Blood Transfusion (NCA). These NHS institutions are each managed by the Blood Service, NHS Blood and Transplant (NHSBT) and both organisations have an ongoing clinical improvement relationship with all UK hospitals. The Clinical Research department of NHSBT assessed this research as not requiring formal NHS ethical approval via the Research Ethics Committee. The hospital visits were carried out accompanied by local staff, who had obtained appropriate approval in each institution. The authors have an agreement to use fully anonymised data from SHOT and NCA for research purposes. We used a question designed to discover adaptations, which was based on previous studies in a hospital dispensary [Sujan et al., 2011a, b]. An additional question was used to assess whether the management or other colleagues found the adaptation to be beneficial. Real-time data collection was undertaken with employees being questioned while performing each of the steps of the transfusion process. A total of 37 staff across the three hospitals were recruited for interviews. All those approached agreed voluntarily to be interviewed and no one declined the request. There was an element of stratification in the selection process. We purposefully aimed to recruit a variety of both clinical and laboratory staff covering each of the nine stages of the transfusion process. At the same time, our sampling was from amongst the staff working in the department during our visit. The staff members interviewed include a variety of healthcare professions such as doctors, nurses, midwifes and scientists, plus ancillary workers such as phlebotomists, administrative staff, porters and healthcare assistants.

\subsection{Data gathering and analysis}

Participants were asked two questions:

Q1: "Please give a short outline of the biggest or most recent difficulty that you have faced when carrying out this procedure and what did you do about the issue?"

Q2: "How supportive was your manager/department for how you solved the issue?" 
The first question, including an open response format, was designed to encourage staff to volunteer their own narrative and to explain any adaptation(s) used to overcome the problem. The second question used a Likert scale and a response format that ranged from 5 (very supportive) to 1 (very unsupportive).

\subsection{Analysis framework}

The data from question 1 were thematically analysed based on the Systems Engineering Initiative for Patient Safety 2.0 model (SEIPS 2.0) [Holden et al., 2013b] and we identified (a) the triggers that led to the requirement to adapt and (b) the system adaptations that were made in response to the triggers.

The categories used were:

- Person(s) - Staff-related factors such as knowledge, education, needs and motivation of people in the system

- Tasks - Specific actions within the process

- Processes - procedures within the larger system

- Tools/Technology (IT) - Information Technology (IT) is a major tool used in healthcare

- Tools/Technology (Non-IT) - Objects used to do work or that assist in doing work, that do not relate to Information Technology (IT)

- Organisation/management - Management control of time, space, resources, activity etc.; organisational culture

- Internal environment - Physical e.g. light, noise, vibration, temperature, physical layout, available space, air quality

- External environment - Factors outside an organisation, e.g. high-level societal, economic, ecological, policy etc.

These categories were applied to investigate both the cause of the triggers leading to a need to adapt and whether the system adaptations were made in the same aspect of the system as the initial trigger, or elsewhere. The permanence status of each adaptation described was also analysed to assess 
if the adaptations were permanent or temporary changes to the system which would indicate whether they were proactive planned improvements or local workarounds.

Answers to question 2 were evaluated to indicate whether managers or colleagues were supportive. We wanted to find out if there were instances of a disconnect between management and front-line staff, so that managers might not be aware of local adaptations. We also aimed to discover whether this separation of management from work-as-done, might result in managers either being unsupportive of certain adaptations, even though they are increasing resilience for the workers, or tacitly approving adaptations.

\section{Findings}

A total of 66 adaptations were identified for analysis from the 37 participants, because, some individuals gave more than one example. Adaptations were seen at every stage of the nine-step transfusion process (Figure 1).

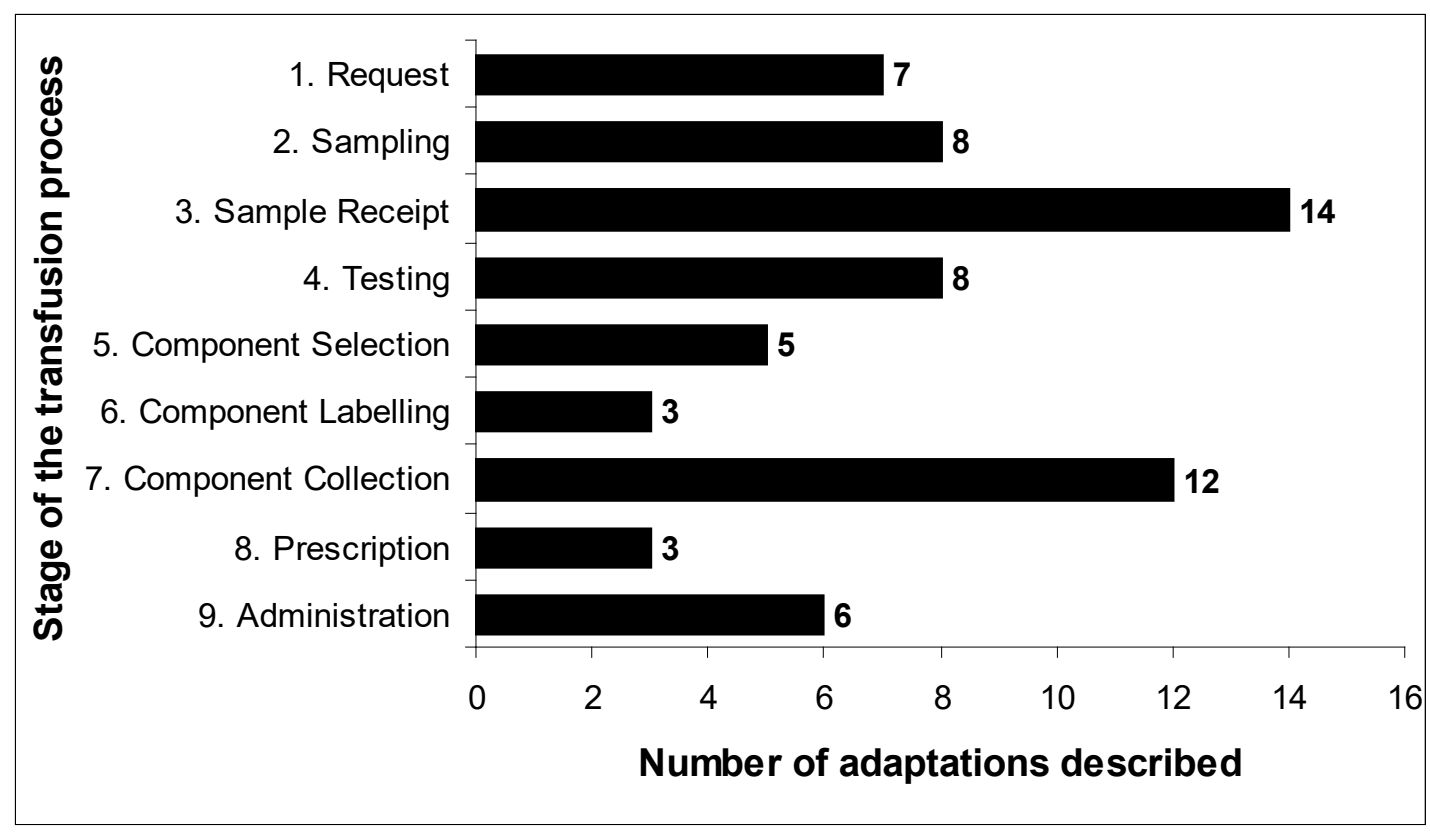

Figure 1: The nine steps in the vein to vein transfusion process [BoltonMaggs et al, 2014] and the number of adaptations at each stage 
3.1 Triggers and the resultant system adaptations that were made to solve the problems

The triggers leading to disturbances in the system can be multifaceted, as can the system adaptations made to resolve these issues. Table 1 shows the quantitative analysis of the narratives, six of which are described below (Example 1 to 6). The largest cause of adaptations is the need to compensate for person-related issues, particularly difficulties with staffing, including not only lack of staff, but situations where employing less qualified or insufficiently trained and experienced staff impacts other colleagues who need to adjust for the deficits (Example 1). Other necessary adaptations related to problems such as deficiencies in Information Technology (IT) or issues with the internal environment and facilities (Example 2). We found no adaptations that were the result of external environment issues, although it could be argued that high-level financial constraints on the United Kingdom (UK) National Health Service (NHS) are the root cause of many of the triggers described.

\begin{tabular}{|c|c|c|c|c|c|c|c|c|}
\hline \multirow{3}{*}{$\begin{array}{l}\text { Adaptation } \\
\text { triggered by }\end{array}$} & \multicolumn{8}{|c|}{ Adaptation made through } \\
\hline & \multirow{2}{*}{ Person(s) } & \multicolumn{2}{|c|}{ Tools/technology } & \multicolumn{2}{|c|}{ Task/process } & \multirow{2}{*}{$\begin{array}{c}\text { Internal } \\
\text { environment }\end{array}$} & \multirow{2}{*}{$\begin{array}{l}\text { Organisation/ } \\
\text { management }\end{array}$} & \multirow{2}{*}{ Totals } \\
\hline & & IT & Non-IT & $\begin{array}{l}\text { Small } \\
\text { task }\end{array}$ & $\begin{array}{l}\text { Large } \\
\text { process }\end{array}$ & & & \\
\hline Person(s) & 2 & 4 & 0 & 5 & 12 & 0 & 0 & 23 \\
\hline $\begin{array}{c}\text { Tools/techn- } \\
\text { ology (IT) }\end{array}$ & 1 & 3 & 1 & 5 & 5 & 0 & 0 & 15 \\
\hline $\begin{array}{l}\text { Tools/techn- } \\
\text { ology (Non-IT) }\end{array}$ & 0 & 1 & 3 & 2 & 2 & 0 & 0 & 8 \\
\hline $\begin{array}{c}\text { Task } \\
\text { (small task) }\end{array}$ & 0 & 0 & 0 & 4 & 0 & 0 & 0 & 4 \\
\hline $\begin{array}{c}\text { Process } \\
\text { (large process) }\end{array}$ & 0 & 1 & 0 & 0 & 6 & 0 & 0 & 7 \\
\hline $\begin{array}{c}\text { Internal } \\
\text { environment }\end{array}$ & 0 & 1 & 2 & 2 & 0 & 2 & 0 & 7 \\
\hline $\begin{array}{l}\text { Organisation/ } \\
\text { management }\end{array}$ & 0 & 0 & 0 & 0 & 2 & 0 & 0 & 2 \\
\hline Totals & 3 & 10 & 6 & 18 & 27 & 2 & 0 & 66 \\
\hline
\end{tabular}

Shaded cells $=$ adaptation in the same part of the system as the trigger $(20 / 66,30.3 \%)$

Table 1: Comparison of the initial adaptation triggers and adapted elements 
Example 1: Adaptation due to lack of staff training/experience: The blood component irradiation practice was adapted from targeted components only to all components in stock being irradiated for a ward that has many immunocompromised patients. Irradiation is needed to protect immunocompromised patients, but due to lack of staff training, clinical staff are not well trained or experienced enough to know when to request irradiated units. Therefore, all blood components for this ward are irradiated. It is a waste to use components with extra specifications for patients that do not require them, but this adaptation has been made to reduce the risk to patients when their clinicians do not know they should be requesting irradiated components.

Example 2: Compensating for environment/facilities problem: The air conditioning in the transfusion laboratory does not work very well and does not control the temperature efficiently. It is a major organisational failure that the temperature problems cannot be corrected, but this is not uncommon, and many hospital areas have to manage with inappropriate temperatures. In particular, the computers situated near the windows are at risk of overheating in direct sunlight. Their only feasible adaptation is to keep the window blinds closed throughout the summertime.

Table 1 also shows that the mechanisms for adaptations did not always occur in the same part of the system as the trigger. It was principally the process that was amended $(n=27)$ or adaptations for a specific task were made $(n=18)$. This illustrates how adaptations are generally carried out within the sphere of influence of staff members. Amending other aspects, such as organisation and management issues or technology and tools, may be outside their control, often due to financial constraints (Example 3). The difficulties that were addressed by adapting the same part of the system as the trigger were less than a third (20/66, 30.3\%). In contrast, over two-thirds of adaptations $(46 / 66,69.7 \%)$ amended a different part of the system (Table 1). Changing a different part of the system may be a less desirable adaptation, but not necessarily less resilient. However, even when adaptations are made in the same aspect, they are not always the most ideal solution, because of wider limitations, such as resource constraints (Example 4).

Limited resources mean that healthcare might not be able to achieve characteristics found in high reliability organisations (HRO) such as extensive training and redundancy [Jeffcott et al., 2009]. Failures have been described as an inability of the system to adapt to disturbances given finite resources 
[Madni and Jackson, 2009] and thus adaptation involves the ability to modify through reconfiguration of available capacity and resources. Madni and Jackson [2009] explain that trade-offs are made to accommodate sustained resource pressure, so organisational resource constraints can potentially magnify exposure to risk. Additionally, it has been shown that hoarding resources (e.g. from a common pool) can create an extra margin locally, but at the expense of other areas that need the resources that are being accumulated [Stephens et al., 2011]. Stephens and colleagues defined that resources could be treated in three ways: defensively to restrict actions of others, autonomously by reorganising to create new margin, or cooperatively by working with others to maximise common pool resources. Studies of common pool resource management suggest that polycentric controls can balance these extremes to create resilient systems [Ostrom, 1999].

Example 3: Simple and cost-effective technology adaptation: Ward staff that collect blood components for their patients from the laboratory refrigerator often need assistance, but the blood storage refrigerator is in a separate room, so laboratory staff cannot keep an eye on the clinical staff. Therefore, a simple two-way baby monitor has been installed to enable laboratory staff to hear what is going on in the collection room and intervene if needed. Each party can communicate via this monitor as required.

Example 4: Adaptation in same part of system but showing financial constraints: Outpatients come in for regular transfusions that take place while sitting in a chair instead of a hospital bed, but this could be problematic if a patient collapsed, which is a risk during transfusion. Resuscitation needs the patient to be lying flat, so the only option may be the floor. Therefore, they have purchased chairs that can easily be made into a flat bed for resuscitation. However, these are expensive and they do not have the budget to replace them all, so some patients are still transfused sitting in chairs that cannot be laid flat for resuscitation.

\subsection{Status of the adaptations: were they permanent or temporary?}

We analysed the status of adaptations to identify how permanent or temporary they were, including whether the permanent adaptations were proactive planned improvements (Figure 2). A minority of adaptations (11/66, 16.7\%) were deliberate adjustments aimed at developing the system and these can be interpreted as quality improvements designed to enhance the process, in comparison to the other permanent process changes (13/66, 19.7\%) which were forced adaptations, i.e. the ideal solution was not going to be available in 
the foreseeable future, so a less than perfect permanent adaptation was made. Most adaptations were provisional changes, either semi-permanent, interim adaptations $(21 / 66,31.8 \%)$, made while awaiting a more ideal solution (Example 5); or an ad hoc, temporary local workaround $(21 / 66,31.8 \%)$ that had become custom and practice for a department or an individual (Example $6)$.

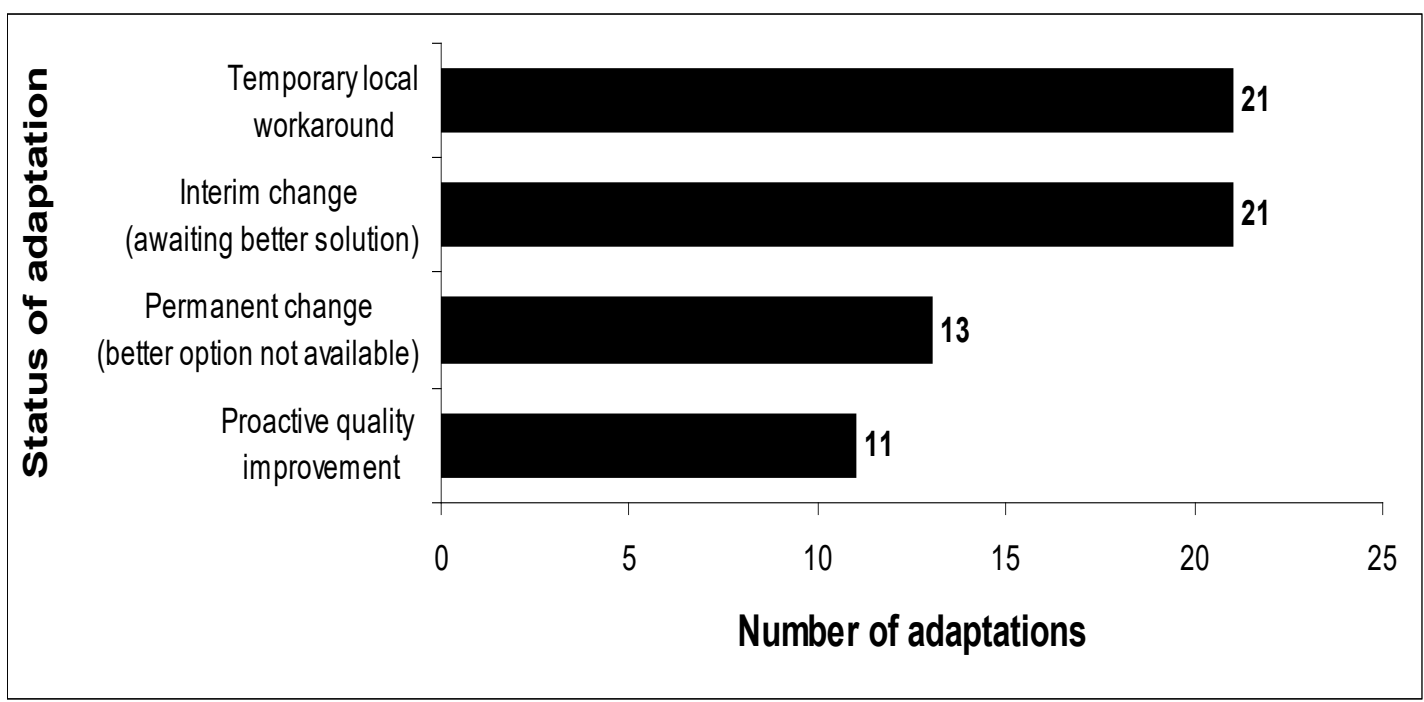

Figure 2: Status of adaptations; permanent or temporary

Example 5 - Interim process change while awaiting IT amendment In the electronic system to order components for transfusion, the possible reasons for transfusion are listed alphabetically, which is less efficient than listing by frequency of use. Therefore, many requests give the reason as 'anaemia', which is one of the earliest alphabetical reasons. Anaemia simply means lacking enough red cells, which is meaningless without further information e.g. is the underlying cause a disease, like cancer, or a massive bleed? Laboratory staff have to phone for more information on every anaemia request to ensure the patient receives appropriate blood components. IT system changes can only be done by the manufacturer, so they cannot amend this themselves.

\section{Example 6 - Temporary local workaround not likely to be resilient} Patients who are transfusion-dependent attend as day-patients, usually at regular 3 to 4 weekly intervals. They need to have a blood sample taken at a specific time in advance of the required transfusion. The timing is critical, because regularly transfused patients need careful monitoring and it can be difficult to match the correct blood for these patients. The nurse who manages the outpatient transfusion clinic assesses a patient's need for transfusion and posts a request form allowing the sample to be taken by the patient's district nurse on the correct day in advance of the planned transfusion. The standard 
process is for the letter to be sent via the hospital administration unit, but this causes delays and the patient may miss their slot. For urgent cases the nurse buys her own stamps to speed up the process. This is not an effective adaptation, because it has not resolved the underlying system problem and relies on the goodwill of one individual.

\subsection{Supportiveness of manager/colleagues for adaptations}

By far the most common response for question 2, "How supportive was your manager/department for how you solved the issue?" was not applicable (N/A), (52/66, 78.8\%). This shows that adaptations were commonly made without the involvement of management or departmental colleagues. Fourteen people (21\%) who responded to this question tended to be very positive with ten people scoring 5 (very supportive). Therefore, good support from managers and/or colleagues may act as a simple local assessment for the acceptability of adaptations.

\section{Discussion}

\subsection{Summary of findings}

We found that adaptations were often forced upon the employees by unforeseen issues and the two largest triggers requiring staff to adapt were staff-related issues and poorly designed health Information Technology (IT) systems that could not be amended or redesigned in a timely manner, if at all (Table 1). These two types of trigger accounted for over a half of all adaptations (38/66, 57.6\%). In comparison, the most common adaptations made were changes to the individual task, or larger process $(45 / 66,68.2 \%)$. Therefore, it seems staff are often unable to resolve the cause of the disturbance to the system, i.e. they do not have the power to improve staffing or amend IT, so they have to adapt elsewhere in order to make the system workable. This finding may also relate to an inclination to 'find and fix', i.e. to adapt in order to resolve a problem, then potentially forget about it. Other research has defined three levels [Jeffs et al., 2012]: (1) 'doing a quick fix' with no further action; (2) 'going into a black hole' reporting problems, but receiving no feedback and (3) 'closing off the Swiss-cheese holes', in which the reported problem is corrected at an organisational level. It has been shown that generally healthcare providers do not prioritise reporting if a safety 
problem is fixed [Hewitt et al., 2015], thus removing the opportunity to learn lessons and possibly resolve the difficulty at management level. This is borne out by responses to question 2 that showed 52/66 (78.8\%) where managers or colleagues were unaware of the adaptations and $39 / 52(59.1 \%)$ showed changes made in a different part of the process to the underlying difficulty. Of these, $28 / 39(71.8 \%)$ had a trigger related to either IT or person issues. These two difficulties are often problematic to resolve at organisational level, so employees accurately assess that their pleas for more resources are unlikely to succeed if it is management policy to cut finances for IT and staff. Therefore, they adapt elsewhere and do not report the actual problem. Example 1 demonstrates this, because the staff deficiencies being resolved by the adaptation (all blood components irradiated) are as a result of a policy to reduce staff training.

Through analysing the permanence status of adaptations, we managed to reveal a spectrum of changes, including deliberately planned quality improvements that were designated as proactive adaptations (Figure 2). The range of adaptations continued with three types of forced changes that also showed a scale of different levels of permanence. These comprised (1) Permanent adaptations that were done when it was accepted that more ideal solutions were not going to be available; (2) Interim changes while awaiting permanent and hopefully better solutions and (3) Temporary, ad hoc workarounds that were typically localised changes. Thus, assessment of the permanence status of the adaptation gave an indication of whether the adaptation would be a long-term success or was acting as a sticking-plaster, (i.e., a short-term workaround). Forced adaptations showed the work system may be deficient and staff felt the need to adapt to get work done. These forced adaptations may not be advantageous and could be unsafe. The shared-learning opportunities from these are limited, but they may be valuable indicators at a local level of a system that is liable to fail, i.e. a potential future accident/incident could be prevented. In contrast, the adaptations defined as proactive changes resulted in a development of the process, often to improve quality and well-being. These positive adaptations usually happened in environments where the surrounding system was adequate and such 
adaptations could be good practices with lessons worth sharing. However, successful adaptations or adjustments that are incorporated into standard procedures become part of work-as-imagined and will then be subject to adaptations and adjustments themselves [Anderson et al., 2016].

The Likert-scale second question was designed to reveal the level of managerial or departmental support for the adjustments being made. However, the high number of N/A scores $(52 / 66,78.8 \%)$ may demonstrate there was insufficient endorsement, often because managers and colleagues were not made aware of changes implemented. Characteristically the reason for this shortcoming was because the staff making adaptations either had no confidence that raising the problem with others, especially management, would result in any improvement, or they were aware that they were deliberately not following standard processes, hence were breaking the rules. Good support, e.g. those scoring $5(n=10)$ may point to the adaptation being suitable and hence increasing resilient abilities. Poor support, e.g. score 1 $(n=2)$ could signify managers or colleagues have concerns about the safety consequences of the adjustment made to the standard process. Underresourcing of the UK NHS means adaptations can result from an acceptance that requesting more staff, training or equipment is not realistic. Managers or senior staff were more likely to have the power to make potentially resilient changes, because they had the necessary authority.

\subsection{Adaptations as a response to the complexity of healthcare} Healthcare is commonly termed a complex socio-technical system, but has also been described as a complex adaptive system [Braithwaite et al., 2013], which inevitably leads to repeated adjustment, so adaptations are not uncommon, but are normal adjustments that people make in order to carry out their routine work. Human adaptability in work systems has been recognised for decades [Rasmussen and Jensen, 1974] and the relationship between adaptation and error is well documented [e.g. Rasmussen, 1990] as is the tendency for human behaviour to migrate towards the boundary of acceptable performance [Rasmussen, 1997]. Therefore, design of work systems should not be based on commonly used methods and tools such as task analysis 
alone, but need to sustain adaptation by acknowledging that conditions cannot always be predicted [Rasmussen, 2000; Hopkins, 2019].

Adaptations to healthcare IT have been well documented in the past [Holden et al. 2013a; Novak et al., 2013] and we found IT problems to be a major reason for making system changes. The ubiquity of IT workarounds is an ongoing issue affecting safe healthcare, because poorly designed IT is often introduced to healthcare [Wears et al. 2014], which forces staff to adapt by forgoing IT security in order to get their work done. Wears et al. highlight that "People are the most adaptable element in any complex work system" and identifies this as a 'tragedy', because the adaptability of staff within a dysfunctional system will paper over the cracks and give a false impression of equilibrium. The general lack of resources in healthcare may lead to inadequate systems and because staff in healthcare are affected by changeable work system elements, they may be more likely to react to and adapt the work system [Carayon et al., 2014].

Adaptations also demonstrate the difficulties involved in healthcare delivery, in this case trying to walk the line between rule compliance and complete empowerment or freedom to carry out a task. There may not be a requirement to prioritise one over the other, as each task needs to be reviewed to decide what is best for that part of the work system [Hale and Swuste, 1998; Hale and Borys, $2013 \mathrm{a}, \mathrm{b}]$. Transfusion is a highly regulated field that does not appear to give staff much freedom. As an illustration there are over 250 international guidelines governing transfusion procedures [ISBT, 2019] compared to 128 international safety standards in the nuclear industry [IAEA, 2019], which is often identified as a high reliability organisation and one which healthcare institutions aim to emulate [Health Foundation, 2011]. Adaptations demonstrate the tensions involved in balancing risk and safety and are suggestive of what Hollnagel [2009] terms the Efficiency-Thoroughness Trade-Off (ETTO) principle, which has been described as a way to characterise the adjustments that are an essential part of everyday performance in complex socio-technical systems, such as healthcare. The adaptation described in Example 1 demonstrates the ETTO principle. It is safe 
to give irradiated blood to any patient, but it is less efficient (additional irradiation step, unnecessary for some) and more costly since the irradiation process reduces the shelf-life of blood components, which requires the need to produce more components to compensate for the reduced shelf-life.

However, this policy tends to err on the side of thoroughness for a specialist ward, as it removes the risk of poorly trained staff giving non-irradiated components to immunocompromised patients.

\subsection{Enhanced Concepts for Applying Resilience Engineering (CARE) model}

Based on our findings of triggers and types of adaptations, we propose an enhanced CARE model (Concepts for Applying Resilience Engineering) as shown in Figure 3, [Anderson et al., 2016]. The CARE model was developed to provide a framework for studying organisational resilience and illustrates the potential for the same adaptive processes to have either acceptable or unacceptable outcomes. 'Work-as-imagined' is planned with an intention to achieve an alignment between demand and the capacity, but 'work-as-done' inevitably faces misalignments between demand and capacity and incorporates the adjustments needed to accommodate the gap. In this study, we probed deeper into the ways adjustments/adaptations were triggered and made. The SEIPS 2.0 model helped categorise both the triggers of adaptations and the various system components adapted.

These additional details on the adaptation triggers and mechanisms can provide additional support for studying organisational resilience. 


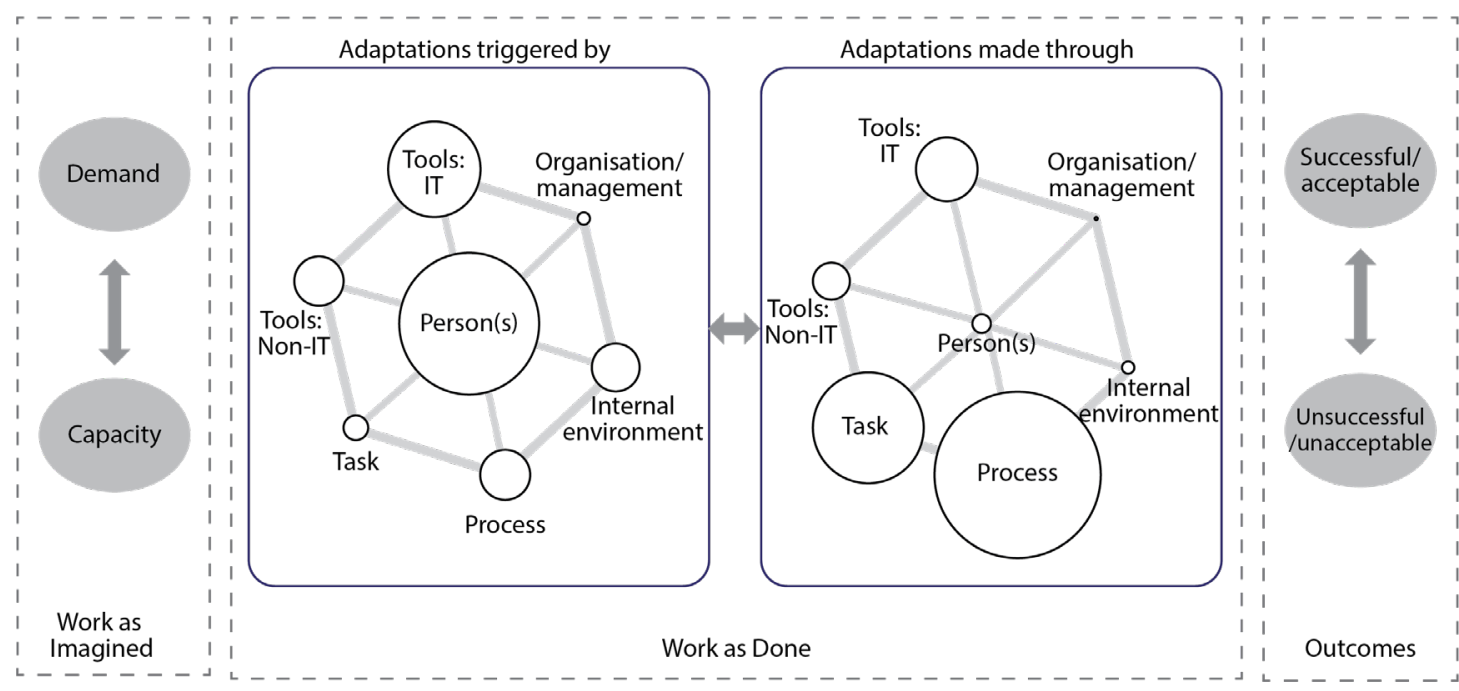

Figure 3: An Enhanced CARE model embracing the adaptation

mechanism (the size of the circles in the Work as Done box are intended to correspond to the data in Table 1)

Figure 3 uses scaled circles to demonstrate the disparity visually between the system components where triggers for adaptation are most common (i.e., staff-related issues and IT problems), and where adaptations actually occur, (i.e., task and process aspects of the system) in blood transfusion. Different parts of healthcare or industries might have different types of system components more prominent in adaptation triggering and mechanism (different size of the circle), but this enhanced CARE model embracing a part of the SEIPS can help to develop an in-depth understanding of the organisational resilience. The in-depth understanding of adaptations may not be appreciated by management, but it can provide examples of good practice to be widely shared. The newly proposed framework may have the potential to build on work already underway in the area of learning from near-miss event analysis as well [Anonymised].

There are problems with standard clinical audits of healthcare processes, which may not be meaningful, as they are not designed to discover or understand the many adaptations staff make to deal with dynamic healthcare problems. Traditional clinical audits, plus the recurrent regulatory audits carried out by the Care Quality Commission (CQC) and the Medicines and Healthcare products Regulatory Agency (MHRA), promote a situation where 
staff are expected to work in a regimented fashion and are only observed for their adherence to standard operating procedures (SOP) and evidence-based guidelines. Audits become a punitive measure, designed to identify where staff are deviating from prescribed practice and therefore audits do not promote possible learning from adaptations. As demonstrated in this study, the simple two questions on adaptations can be used and introduced as a non-threatening learning review, forming a proactive part of the regular local clinical audits. This could encourage staff to assess their own adaptations in an ongoing manner and amend standard processes dynamically. Our healthcare resilience audit method can lead to benefits similar to the observational work done in air traffic management (ATM), i.e. Normal Operations Safety Survey (NOSS) [Skybrary, 2016] and the aviation audit procedure that uses trained observers in normal flights, Line Operations Safety Audit (LOSA) [Skybrary, 2017].

Finally, analysis using the modified CARE model shows that different attempts to manage the misalignment between demand and capacity resulted in varying levels of successful or unsuccessful adaptation. This can be demonstrated by some of the specific examples described earlier. Example 3 can be considered as a successful adaptation, which uses a simple commercial product (a baby monitor) to provide the extra capacity needed to match the demand created by inexpert staff carrying out a task in a separate room without direct supervision. Conversely, example 5 describes an adaptation that is reducing capacity by adding an extra step to the process (making a phone call) as a result of an inefficient computer programme. This example shows the importance of having adaptation sharing/feedback mechanisms with other people in the system to improve patient safety. This demonstrates there is a problem with any culture that blames individuals for an incident that occurs after amending a standard process [Dekker, 2012], because that amendment might have previously been necessary and successful on numerous occasions. An understanding of how to assess the resilience of adaptations should improve the safety of healthcare systems more than concentrating on the improving the resilience of individuals within the system [Balme et al., 2015, Oliver 2017]. 


\section{Conclusions}

This study set out to examine the extent and nature of adaptations at all stages of the full vein to vein transfusion process. The major finding is that triggers for adaptations were typically staff-related, such as understaffing or insufficient knowledge and training, or resulting from problems with tools and technology, particularly inflexible information technology (IT). However, staff are often unable to resolve the source of the difficulty, so are forced to adapt elsewhere in the system and make changes within their sphere of influence. Therefore, the most common means of adapting are in task and process areas, because individual staff members cannot often influence the root causes of the problems. Managers and colleagues may not be made aware of changes and this could reduce the chances of improving the overall system resilience and safety. Based on the insights gained from the findings, an enhanced CARE model (embracing a part of the SEIPS model for the in-depth analysis of adaptations) was proposed to help in studying organizational resilience.

This study has demonstrated that two simple questions on adaptations and the enhanced CARE framework can provide in-depth insights into work-asdone in blood transfusion. This understanding of work-as-done can help achieving a meaningful system analysis and improvement. This approach can be scaled up to become part of a nationwide vein to vein audit of all transfusion procedures in order to assess resilience. In addition, a similar approach could be employed in clinical audits in other fields to draw attention to adaptations being made by people at the sharp end in any healthcare field.

A limitation of this study is the reliance on interview. The data gathering exercise assumes the staff interviewed gave candid responses. Although there is no reason to believe they were not open with their replies, this might be affected by their level of confidence that their organisation has a no blame or just culture [Dekker, 2012]. The analyses in the results section are often reliant on subjective categorisations, which was minimised by the checks by multiple researchers. 
Further work is planned, including gathering more data during a large 'Vein to Vein Audit' (V2V), which is being carried out as part of the programme for the National Comparative Audit of Blood Transfusion [NCA, 2018]. This will be a continuous voluntary audit available to all hospitals throughout the UK. The V2V audit will cover traditional audit observations of the standard procedures at all nine steps of the transfusion process, but for each step the two questions asked in this research study will also be included. Analysis of answers given to these two questions during the V2V audit will show whether this type of resilience audit can be scaled up and carried out by local clinical audit staff. It is anticipated that this audit may highlight areas of the transfusion process where adaptations could be made to reduce patientsafety incidents or where learning could be shared about existing successful adjustments or changes.

\section{Acknowledgements}

Anonymised.

Word count $=6105$ (excl. figures and tables) 


\section{References}

Acher, A.W., LeCaire, T.J., Hundt, A.S., Greenberg, C.C., Carayon, P., Kind, A.J. and Weber, S.M., 2015. Using human factors and systems engineering to evaluate readmission after complex surgery. Journal of the American College of Surgeons, 221(4), pp.810-820. doi: 10.1016/j.jamcollsurg.2015.06.014

Anderson, J.E., Ross, A.J., Back, J., Duncan, M., Snell, P., Walsh, K. and Jaye, P., 2016. Implementing resilience engineering for healthcare quality improvement using the CARE model: a feasibility study protocol. Pilot and Feasibility Studies, 2(1), p.61. doi: 10.1186/s40814-016-0103-x.

Balme, E., 2015. Doctors need to be supported, not trained in resilience. BMJ: British Medical Journal, 351, p.h4709. doi: 10.1136/bmj.h4709.

Bolton-Maggs, P.H.B. (Ed), Poles et al. on behalf of the Serious Hazards of Transfusion (SHOT) Steering Group, 2014. The 2013 Annual SHOT Report [Online]. Available at <https://www.shotuk.org/> [accessed 27 January 2019].

Bolton-Maggs, P.H.B. (Ed), Poles et al. on behalf of the Serious Hazards of Transfusion (SHOT) Steering Group, 2018. The 2017 Annual SHOT Report [Online]. Available at <https://www.shotuk.org/> [accessed 27 January 2019].

Braithwaite, J., Clay-Williams, R., Nugus, P. and Plumb, J., 2013. Health care as a complex adaptive system. In: Hollnagel, E., Braithwaite, J. \& Wears, R. (eds.) Resilient Health Care. Ashgate Publishing Limited, Farnham, Surrey, pp.57-73.

Carayon, P. (Ed.), 2011, Handbook of human factors and ergonomics in health care and patient safety. Boca-Raton: CRC Press.

Carayon, P., Wetterneck, T.B., Rivera-Rodriguez, A.J., Hundt, A.S., Hoonakker, P., Holden, R., Gurses, A.P., 2014. Human factors systems approach to healthcare quality and patient safety. Applied Ergonomics, 45(1):14-25. doi: 10.1016/j.apergo.2013.04.023.

Commission on Education and Training for Patient Safety, 2016. Improving safety through education and training, Health Education England, Leeds, https://www.hee.nhs.uk/our-work/education-training-patient-safety [accessed 27 January 2019].

CQC, 2018. Care Quality Commission, Quality improvement in hospital trusts: sharing learning from trusts on a journey of QI. 2018.

https://www.cqc.org.uk/publications/evaluation/quality-improvement-hospitaltrusts-sharing-learning-trusts-journey-qi [accessed 27 January 2019].

Dekker, S., 2012. Just Culture: Balancing Safety and Accountability. Ashgate Publishing, Ltd.

GMC, 2018. Human Factors training to be rolled out for investigators [Online]. Available at <https://www.gmc-uk.org/news/news-archive/human-factorstraining-to-be-rolled-out-for-investigators> [accessed 27 January 2019].

Hale, A.R. and Swuste, P.H.J.J., 1998. Safety rules: procedural freedom or action constraint? Safety Science, 29(3), pp.163-177. doi: 10.1016/S09257535(98)00020-4. 
Hale, A.R. and Borys [2013a]. Working to rule or working safety? Part 1: A state of the art review. Safety Science, 55, 207-221. doi:

10.1016/j.ssci.2012.05.011.

Hale, A.R. and Borys [2013b]. Working to rule or working safety? Part 2: The management of safety rules and procedures. Safety Science, 55, 222-231. doi: 10.1016/j.ssci.2012.05.013.

Health Foundation (2011) Evidence Scan: High Reliability Organisations. Health Foundation, London [Online]. Available at <https://www.health.org.uk/sites/default/files/HighReliabilityOrganisations.pdf> [accessed 27 January 2019].

Hewitt, T.A. and Chreim, S., 2015. Fix and forget or fix and report: a qualitative study of tensions at the front line of incident reporting. BMJ Qual Saf, 24(5), pp.303-310. doi: 10.1136/bmjqs-2014-003279.

Holden, R.J., Rivera-Rodriguez, A.J., Faye, H., Scanlon, M.C. and Karsh, B.T., 2013a. Automation and adaptation: nurses' problem-solving behavior following the implementation of bar-coded medication administration technology. Cognition, Technology \& Work, 15(3), pp.283-296. doi: 10.1007/s10111-012-0229-4.

Holden, R.J., Carayon, P., Gurses, A.P., Hoonakker, P., Hundt, A.S., Ozok, A.A. and Rivera-Rodriguez, A.J., 2013b. SEIPS 2.0: a human factors framework for studying and improving the work of healthcare professionals and patients. Ergonomics, 56(11), pp.1669-1686. doi:

10.1080/00140139.2013.838643.

Hollnagel, E. and Goteman, O., 2004. The functional resonance accident model. Proceedings of Cognitive System Engineering in Process Plant, 2004, pp.155-161.

Hollnagel, E., 2009. The ETTO Principle: Efficiency-Thoroughness Trade-Off. Why things that go right sometimes go wrong?. Farnham (UK): Ashgate. doi: $10.1201 / 9781315616247$.

Hollnagel, E., 2012. Functional Resonance Analysis Method: Modelling complex sociotechnical systems. Ashgate, Farnham, UK.

Hollnagel, E., 2014. Safety-I and safety-II: The past and future of safety management. Ashgate Publishing, Ltd.

Hollnagel, E. 2015. Why is work-as-imagined different from work-as- done? In Wears, R. L., Hollnagel, E., \& Braithwaite, J. (Eds.). Resilient Health Care, Volume 2: The Resilience of Everyday Clinical Work (pp. 249-264). Ashgate Publishing, Ltd.

Hopkins, A. 2019. Organising for Safety - How Structure Creates Culture. Sydney: Wolters Kluwer CCH Australia.

HSIB, 2018. Healthcare Safety Investigation Branch Annual Review 2017/18 [Online]. Available at <https://www.hsib.org.uk/documents/46/HSIB Annual Review July 2018 ol w8VVx.pdf> [Accessed 27 January 2019]. 
IAEA, 2019. International Atomic Energy Authority, Safety Standards [Online]. Available at $<$ https://www.iaea.org/resources/safety-standards $>$ [Accessed 27 January, 2019].

Infected Blood Inquiry, 2019. Explore the Enquiry [Online]. Available at $<$ https://www.infectedbloodinquiry.org.uk/> [Accessed 27 January 2019].

ISBT, 2019. International Society of Blood Transfusion, Guidelines [Online]. Available at <ISBT https://education.isbtweb.org/> [Accessed 27 January, 2019].

Jeffcott, S.A., Ibrahim, J.E. and Cameron, P.A., 2009. Resilience in healthcare and clinical handover. BMJ Quality \& Safety, 18(4), pp.256-260. doi: 10.1136/qshc.2008.030163.

Jeffs, L., Berta, W., Lingard, L. and Baker, G.R., 2012. Learning from near misses: from quick fixes to closing off the Swiss-cheese holes. BMJ Qual Saf, 21(4), pp.287-294. doi: 10.1136/bmjqs-2011-000256.

Kirwan, B. and Shorrock, S.T., 2014. A view from elsewhere: safety culture in European air traffic management. In: Waterson, P. (Ed). Patient Safety Culture: Theory, Methods and Application. Ashgate Publishing, Ltd.

Madni, A.M. and Jackson, S., 2009. Towards a conceptual framework for resilience engineering. IEEE Systems Journal, 3(2), pp.181-191. doi: 10.1109/JSYST.2009.2017397.

Nakajima, K., 2015. Blood transfusion with health information technology in emergency settings from a Safety-II Perspective. In Wears, R. L., Hollnagel, E., \& Braithwaite, J. (Eds.). Resilient Health Care, Volume 2: The Resilience of Everyday Clinical Work (pp. 99-113). Ashgate Publishing, Ltd.

NCA, 2018. Schedule of Audits [Online]. Available at <http://hospital.blood.co.uk/audits/national-comparative-audit/> [accessed 27 January 2019].

NHS England, 2013. Human Factors in Healthcare - A Concordat from the National Quality Board [Online]. Available at $<$ https://www.england.nhs.uk/wpcontent/uploads/2013/11/nqb-hum-fact-concord.pdf> [accessed 27 January 2019].

Novak, L.L., Holden, R.J., Anders, S.H., Hong, J.Y. and Karsh, B.T., 2013. Using a sociotechnical framework to understand adaptations in health IT implementation. International Journal of Medical Informatics, 82(12), pp.e331e344. doi: 10.1016/j.jimedinf.2013.01.009.

Oliver, D., 2017. David Oliver: When" resilience" becomes a dirty word: Acute Perspective. BMJ: British Medical Journal, 358, p.j3604. doi: 10.1136/bmj.j3604.

Ostrom, E., 1999. Coping with tragedies of the commons. Annual Review of Political Science, 2(1), pp.493-535. doi: 10.1146/annurev.polisci.2.1.493.

Pickup, L., Atkinson, S., Hollnagel, E., Bowie, P., Gray, S., Rawlinson, S. and Forrester, K., 2017. Blood sampling-Two sides to the story. Applied Ergonomics, 59, pp.234-242. doi: 10.1016/j.apergo.2016.08.027. 
Rasmussen, J. and Jensen, A., 1974. Mental procedures in real-life tasks: A case study of electronic trouble shooting. Ergonomics, 17(3), pp.293-307. doi: $10.1080 / 00140137408931355$.

Rasmussen, J., 1990. Human error and the problem of causality in analysis of accidents. Phil. Trans. R. Soc. Lond. B, 327(1241), pp.449-462. doi: 10.1098/rstb.1990.0088.

Rasmussen, J., 1997. Risk management in a dynamic society: a modelling problem. Safety Science, 27(2-3), pp.183-213. doi: 10.1016/S09257535(97)00052-0.

Rasmussen, J., 2000, July. Designing to support adaptation. In Proceedings of the Human Factors and Ergonomics Society Annual Meeting (Vol. 44, No. 22, pp. 554-557). Sage CA: Los Angeles, CA: SAGE Publications. doi: $10.1177 / 154193120004402215$.

Reason, J., Parker, D. and Lawton, R., 1998. Organizational controls and safety: The varieties of rule-related behaviour. Journal of Occupational and Organizational Psychology, 71(4), pp.289-304.

SHOT, 1996-2017. Annual SHOT Reports 1996 to 2017 [Online]. Available at <https://www.shotuk.org/> [accessed 27 January 2019].

Skybrary, 2016. Normal Operations Safety Survey (NOSS) in ATM [Online]. Available at

$<$ https://www.skybrary.aero/index.php/Normal Operations Safety Survey (N OSS) in ATM> [accessed 27 January 2019].

Skybrary, 2017. Line Operations Safety Audit (LOSA) [Online]. Available at $<$ https://www.skybrary.aero/index.php/Line Operations Safety Audit (LOSA) $>$ [accessed 27 January 2019].

Stephens, R.J., Woods, D.D., Branlat, M. and Wears, R.L., 2011, June. Colliding dilemmas: interactions of locally adaptive strategies in a hospital setting. In 4th Resilience Engineering International Symposium.

Sujan, M.A., Ingram, C., McConkey, T., Cross, S. and Cooke, M.W., 2011a. Hassle in the dispensary: pilot study of a proactive risk monitoring tool for organisational learning based on narratives and staff perceptions. Quality and Safety in Health Care, pp.bmjqs-2010. BMJ Quality \& Safety, 20(6), pp.549556. doi: 10.1136/bmjqs.2010.048348.

Sujan, M.A., Pozzi, S., Valbonesi, C. and Ingram, C., 2011b. Resilience as individual adaptation: preliminary analysis of a hospital dispensary. In: Proceedings of HCP 2011-Fourth Workshop on Human Centered Processes.

Sujan, M.A., Huang, H. and Braithwaite, J., 2017. Learning from incidents in health care: Critique from a Safety-II perspective. Safety Science, 99, pp.115121. doi: 10.1016/j.ssci.2016.08.005.

Sujan, M.A., Furniss, D. Anderson, J., Braithwaite, J. and Hollnagel, E. 2019. Resilient Health Care as the basis for teaching patient safety - A Safety-II critique of the World Health Organisation patient safety curriculum. Safety Science, 118, 15-21. doi: 10.1016/j.ssci.2019.04.046. 
Vincent, C. and Amalberti, R., 2016. Safety strategies in hospitals. In Safer Healthcare (pp. 73-91). Springer, Cham. doi: 10.1007/978-3-319-25559-0 https://www.springer.com/gb/book/9783319255576 [accessed 27 January 2019].

Waterson, P.E. and Catchpole, K. 2016, Human factors in healthcare: welcome progress, but still scratching the surface. BMJ: Quality and Safety, 25(7), pp.480-484. doi: 10.1136/bmjqs-2015-005074.

Anonymised reference

Anonymised reference

Wears, R.L. and Hettinger, A.Z., 2014. The tragedy of adaptability. Annals of Emergency Medicine, 63(3), pp.338-339. doi: 10.1016/j.annemergmed.2013.10.035.

Wooldridge, A.R., Carayon, P., Hundt, A.S. and Hoonakker, P.L., 2017. SEIPS-based process modeling in primary care. Applied ergonomics, 60, pp.240-254. doi: 10.1016/j.apergo.2016.11.010. 